Revue

Revue de l'histoire des religions

de Ihistoire des religions

$3 \mid 2012$

Varia

\title{
La condanna del modernismo, Documenti, interpretazioni, conseguenze a cura di Claus ARNOLD e Giovanni VIAN
}

Roma, Viella, 2010, 260 p., 22 cm, ISBN 978-88-8334-440-4

Louis-Pierre Sardella

\section{OpenEdition}

Journals

Édition électronique

URL : http://journals.openedition.org/rhr/7960

DOI : 10.4000/rhr.7960

ISSN : 2105-2573

Éditeur

Armand Colin

Édition imprimée

Date de publication : 1 septembre 2012

Pagination : 460-462

ISBN : 978-2200-92975-0

ISSN : 0035-1423

Référence électronique

Louis-Pierre Sardella, "La condanna del modernismo, Documenti, interpretazioni, conseguenze a cura di Claus arnold e Giovanni vian », Revue de l'histoire des religions [En ligne], 3 | 2012, mis en ligne le 05 octobre 2012, consulté le 22 septembre 2020. URL : http://journals.openedition.org/rhr/7960 ; DOI : https://doi.org/10.4000/rhr.7960

Ce document a été généré automatiquement le 22 septembre 2020.

Tous droits réservés 


\title{
La condanna del modernismo, Documenti, interpretazioni, conseguenze a cura di Claus ARNOLD e Giovanni VIAN
}

Roma, Viella, 2010, 260 p., 22 cm, ISBN 978-88-8334-440-4

\author{
Louis-Pierre Sardella
}

\section{RÉFÉRENCE}

La condanna del modernismo, Documenti, interpretazioni, conseguenze a cura di Claus ARNOLD e Giovanni vIAN, Roma, Viella, 2010, 260 p., 22 cm, ISBN 978-88-8334-440-4

1 Depuis quelques années, à la suite de l'ouverture d'archives jusqu'alors inaccessibles, comme par exemple celles de la Congrégation pour la doctrine de la foi, les études sur le modernisme se multiplient, en particulier en Allemagne et en Italie. C'est ainsi que les professeurs Claus Arnold et Giovanni Vian viennent de rassembler dans un ouvrage collectif six contributions (qui sont pour la plupart la reprise développée d'articles déjà parus), toutes en italien, s'appuyant sur des fonds nouveaux peu ou pas exploités jusqu'à maintenant.

2 L'introduction définit bien les objectifs de l'ouvrage. Il s'agit d'une part d'étudier la crise moderniste en tant que telle, dans sa spécificité propre, comme la tentative de repenser le message chrétien à la lumière des besoins de la société au tournant des XIX ${ }^{\mathrm{e}}$ et $\mathrm{xx}^{\mathrm{e}}$ siècles. De ce point de vue, le modernisme apparaît comme la phase la plus aiguë de la confrontation du catholicisme avec le monde moderne engagé dans un processus de sécularisation touchant l'ensemble du champ social et culturel. Il s'agit d'autre part de mettre en évidence les connexions de la crise moderniste avec des processus de plus longue durée et de détecter les conséquences importantes et durables qu'elle a eues sur les rapports de l'Église avec le monde moderne. De ce point de vue, le modernisme 
apparaît comme un événement non réductible aux seules années du pontificat de Pie X, événement dont il convient de suivre la trace dans l'histoire de l'Église contemporaine dans des contextes et avec des enjeux différents de ceux dans lesquels il est apparu.

Trois contributions s'attachent à des cas individuels : celles d'Annnibale Zambarbieri consacrée aux premières censures visant Ernesto Buonaiuti, de Giacomo Losito analysant les interrogations du Père Laberthonnière sur la manière dont les Annales de philosophie chrétienne devaient rendre compte de l'encyclique Pascendi, enfin de Raffaella Perrin sur les difficultés de Mgr Rodolfi, évêque de Vicence, avec la presse catholique intransigeante de son diocèse. Les trois autres abordent des problèmes institutionnels : Claus Arnold analyse l'élaboration du décret Lamentabili sane exitu; Giovanni Vian s'attache à la réception de l'encyclique Pascendi par les épiscopats d'Italie et de France ; Judith Schepers enfin évoque la question de l'interprétation du serment antimoderniste. Ces regards croisés donnent de la crise moderniste une image complexe tant dans les processus d'élaboration des décisions au sein des Congrégations de l'Index et du Saint Office que dans les diverses modalités de leur réception et de leur interprétation.

4 Sur le premier aspect, le traitement de la crise par le Vatican ne s'est pas fait sans tensions. Claus Arnold, fait l'hypothèse qu'une «fonction de l'anti-modernisme à ses débuts, peut-être trop peu prise en compte, fut celle de servir à cacher les désaccords au sein de la scolastique ». L'analyse qu'il présente des discussions visant à atténuer le caractère abrupt de certaines propositions du décret Lamentabili (1907) et à sauvegarder ainsi la possibilité d'une interprétation ultérieure moins rigoureuse est tout à fait probante. Évitant d'ailleurs le terme de « modernisme », Lamentabili est le résultat d'un compromis entre les diverses tendances qui s'affrontaient à l'intérieur de la Curie romaine, car il n'a pas été possible de trouver au problème de la relation entre l'histoire et le dogme une solution commune et conséquente. C'est pourquoi, conçu à l'origine pour défendre, de manière générale, l'Église de la menace rationaliste, le décret s'est finalement concentré sur l'exégèse de l'abbé Loisy.

C'est une incertitude voisine qui explique les hésitations dans l'interprétation du serment antimoderniste (1910). Très vite en effet on s'est interrogé en pratique sur la nature du serment institué par le Motu proprio Sacrorum Antistitum. Si certains, comme les PP. Billot et van Rossum, entendent lui conférer un caractère comparable à un acte de foi, les cardinaux de la Congrégation écartant de la formule le terme de "professio » limitent la portée à un plan strictement disciplinaire. C'est l'opinion qui a prévalu lorsque s'est posée la question de savoir s'il fallait inclure le serment antimoderniste dans le texte du nouveau code de Droit canonique, car comme le déclare un consulteur, il ne fallait pas « donner un caractère de stabilité et de fermeté perpétuelle, comme on le donne à la profession de la foi, à une condamnation d'erreurs conditionnées par des circonstances temporelles. »

De même, si d'un point de vue formel, les épiscopats d'Italie et de France manifestent une adhésion unanime à l'encyclique Pascendi (1907), il faut noter que des voix s'élèvent, et non des moindres, pour défendre l'idée que l'encyclique est, plutôt qu'une condamnation, un cri d'alarme par lequel Pie $\mathrm{X}$ a «corrigé sagement tant d'idées erronées, dissipé tant d'équivoques déplorables, et fait resplendir lucidement la vérité. » Dans cette perspective, il n'y a pas lieu d'arrêter les études et les recherches, car si Pascendi « indique les écueils (à) éviter pour ne pas sombrer dans l'erreur, tout le reste de la mer reste libre et les intellectuels peuvent la parcourir librement et 
ardemment ", écrit par exemple, le cardinal Capecelatro, archevêque de Capoue. Il n'en demeure pas moins que les très rares prélats qui tentent d'avoir une attitude moins radicale que celle imposée par l'offensive antimoderniste, ne disposent d'aucune marge de manœuvre. On le voit bien dans le cas de Mgr Rodolfi, évêque de Vicence, qui ne reçoit aucun soutien de la part du Saint-Siège dans ses démêlés avec les milieux intransigeants de son diocèse, lesquels dénoncent auprès du cardinal de Laï ses tendances modernistes, alors qu'il ne faisait que défendre, contre les attaques de clercs et de journalistes laïcs, ses prérogatives d'évêque.

7 Ces divergences d'interprétations, cette volonté de maintenir des espaces de liberté, ce souci de ne pas compromettre l'avenir sont autant d'éléments qui se retrouvent, peu ou prou, dans la manière dont les clercs ici étudiés, prennent leurs distances vis-à-vis des orientations pontificales. C'est le cas d'Ernesto Buonaiuti, principal auteur de Il programma dei modernisti et qui ne se sent nullement concerné par l'excommunication fulminée contre les auteurs, au motif qu'une « excommunication contre des anonymes était une contradiction dans les termes ». C'est le cas du P. Laberthonnière présenté comme "un catholique non courtisan" par G. Losito qui met bien en lumière un exemple de fidélité au primat de la conscience croyante refusant tout ce qui pourrait apparaître comme forme de complicité avec une autorité dont on ne partage pas les orientations et moins encore la manière de les imposer. En affirmant que «le catholicisme est autre chose qu'une soumission aux hommes ", l'Oratorien développe une réflexion théorique originale sur l'autorité magistérielle et annonce d'une certaine façon ce qui deviendra un comportement ordinaire de beaucoup de fidèles.

8 L'intérêt de ces études est de montrer que, au-delà du moment spécifique de la crise du début $d u x^{e}$ siècle et de la réaction brutale du Saint-Siège face à une menace qu'il considérait comme potentiellement mortelle, les références au modernisme ont joué comme un réflexe de défense de l'ecclésiologie élaborée au xIX siècle : la condamnation du modernisme, en fermant de fait tout espace à une réflexion théologique prenant en compte les interrogations soulevées par les novateurs, a contribué de manière décisive à figer l'attitude de l'Église catholique à l'égard de la société moderne. Les coordinateurs insistent dans leur introduction sur le fait que la hantise du modernisme a contribué à l'affaiblissement implicite de la doctrine conciliaire de Vatican II et de la vision renouvelée du christianisme qu'elle proposait. Constatant que les références explicites à Pascendi sont plutôt rares, au-delà des années immédiatement consécutives à sa publication, G. Vian estime que les courants de pensée qui en étaient le fondement sont restés actifs dans l'enseignement officiel de l'Église catholique, "sous des formes et avec une fréquence assez élevée encore que largement méconnue, jusqu'au seuil du troisième millénaire, en particulier pour renforcer les liens de la discipline ecclésiastique et pour combattre les tentatives de renouvellement de la théologie cherchant à valoriser l'autonomie de l'homme ».

9 Ce livre, dont il faut souhaiter la rapide traduction en français, conforte l'idée que le moment moderniste constitue un ébranlement majeur dans l'histoire du catholicisme contemporain. Il montre aussi tout l'intérêt de sources jusqu'à présent peu exploitées, comme par exemple les comptes rendus de délibération des Conseils de Vigilance. Il invite enfin les chercheurs à ouvrir de nouveaux chantiers qui permettent d'aller de l'événement à ses traces en resituant dans une problématique longue ce moment crucial de l'histoire de l'Église catholique. 